\title{
Average Entropy of a Subsystem
}

\author{
Siddhartha Sent \\ School of Mathematics, University of Dublin, Trinity College, Dublin, Ireland
}

(November 10, 2018)

It was recently conjectured by D. Page that if a quantum system of Hilbert space dimension $\mathrm{nm}$ is in a random pure state then the average entropy of a subsystem of dimension $m$ where $m \leq n$ is $S_{m n}=\sum_{k=n+1}^{m n}(1 / k)-(m-1) / 2 n$. In this letter this conjecture is proved.

PACS numbers: 05.30.ch,03.65.-w,05.90.+m

In a recent letter Page [1] considered a system $A B$ with Hilbert space dimension $m n$. The system consisted of two subsystems $A$ and $B$ of dimensions $m$ and $n$ respectively. Page calculated the average

$$
S_{m n}=<S_{A}>
$$

of the entropy $S_{A}$ over all pure states $\rho=|\Psi><\Psi|$ of the total system where $S_{A}=-\operatorname{Tr} \rho_{A} \ln \rho_{A}$ and $\rho_{A}$, the density matrix of subsystem $\mathrm{A}$, is obtained by taking the partial trace of the full density matrix $\rho$ over the other subsystem, that is, $\rho_{A}=\operatorname{Tr}_{B} \rho$.

The average was defined with respect to the unitary invariant Haar measure on the space of unitary vectors $\mid \Psi>$ in the $m n$ dimensional Hilbert space of the total system. The quantity $\left(\ln m-S_{m n}\right)$ was used to define the average information of the subsystem A. It is a measure of the information regarding the fact that the entire system is a pure state that is contained in the subsystem $m$. Using earlier work [2] 3] in this area, Page was led to consider the probability distribution of the eigenvalues of $\rho_{A}$ for the random pure states $\rho$ of the entire system. He used

$$
\begin{aligned}
& P\left(p_{1}, \ldots, p_{m}\right) d p_{1} \ldots d p_{m} \\
& =N \delta\left(1-\sum_{i=1}^{m} p_{i}\right) \prod_{1 \leq i<j \leq m}\left(p_{i}-p_{j}\right)^{2} \prod_{k=1}^{m} p_{k}^{n-m} d p_{k}
\end{aligned}
$$

where $p_{i}$ was an eigenvalue of $\rho_{A}$ and the normalisation constant for this probability distribution was given only implicitly by the requirement that the total probability integrated to unity. Page then showed that the average

$$
\begin{aligned}
S_{m n} & =-\int\left(\sum_{i=1}^{m} p_{i} \ln p_{i}\right) P\left(p_{1}, \ldots, p_{m}\right) d p_{1} \ldots d p_{m} \\
& =\psi(m n+1)-\frac{\int\left(\sum_{i=1}^{m} q_{i} \ln q_{i}\right) Q d q_{1} \ldots d q_{m}}{m n \int Q d q_{1} \ldots d q_{m}}
\end{aligned}
$$

where $q_{i}=r p_{i}$ for $i=1, \ldots, m, r$ is positive [1], and

$$
\psi(m n+1)=-C+\sum_{k=1}^{m n} \frac{1}{k},
$$

$C$ being Euler's constant, and

$Q\left(q_{1}, \ldots, q_{m}\right) d q_{1} \ldots d q_{m}=\prod_{1 \leq i<j \leq m}\left(q_{i}-q_{j}\right)^{2} \prod_{i=1}^{m} e^{-q_{i}} q_{i}^{n-m} d q_{i}$.

On the basis of evaluating $S_{m n}$ for $m=2,3,4,5$ using Mathematica 2.0, Page conjectured that the exact result for $S_{m n}$ was

$$
S_{m n}=\sum_{k=n+1}^{m n} \frac{1}{k}-\frac{(m-1)}{2 n},
$$

but was not able to prove that this was the case. In this letter, we will prove this conjecture.

We first observe that the van der Monde determinant defined by

$$
\Delta\left(q_{1}, \ldots, q_{m}\right) \equiv \prod_{i \leq i<j \leq m}\left(q_{i}-q_{j}\right)
$$

may be written

$$
\Delta\left(q_{1}, \ldots, q_{m}\right)=\left|\begin{array}{ccc}
1 & \cdots & 1 \\
q_{1} & \cdots & q_{m} \\
\vdots & \ddots & \vdots \\
q_{1}^{m-1} & \cdots & q_{m}^{m-1}
\end{array}\right|
$$

We next observe that $\Delta\left(q_{1}, \ldots, q_{m}\right)$ can be written as

$$
\Delta\left(q_{1}, \ldots, q_{m}\right)=\left|\begin{array}{ccc}
p_{0}\left(q_{1}\right) & \cdots & p_{0}\left(q_{m}\right) \\
p_{1}\left(q_{1}\right) & \cdots & p_{1}\left(q_{m}\right) \\
\vdots & \ddots & \vdots \\
p_{m-1}\left(q_{1}\right) & \cdots & p_{m-1}\left(q_{m}\right)
\end{array}\right|
$$

for any set of polynomials $p_{k}(q), k=0, \ldots, m-1$, which have the property, $p_{0}(q)=1$, and

$$
p_{k}(q)=q^{k}+C_{k-1} q^{k-1}+\cdots+C_{0}, \quad k=1, \ldots, m-1 .
$$

This immediately follows from the fact that the value of a determinant does not change if the multiple of any one row is added to a different row.

We now choose polynomials $p_{k}^{\alpha}(q)$ judiciously. We introduce orthogonal polynomials $p_{k}^{\alpha}(q)$ with the properties:

$$
\text { 1. } p_{k}^{\alpha}(q)=q^{k}+C_{k-1} q^{k-1}+\cdots+C_{0}^{\alpha}, \quad p_{0}^{\alpha}(q)=1 .
$$




\section{2. $\int_{0}^{\infty} d q e^{-q} q^{\alpha} p_{k_{1}}^{\alpha}(q) p_{k_{2}}^{\alpha}(q)=h_{k_{1}}^{\alpha} \delta_{k_{1} k_{2}}, \quad \alpha=n-m$.}

Polynomials with these properties are well known. They are the generalised Laguerre polynomials defined by $[4$

$$
p_{k}^{\alpha}(q)=\frac{e^{q}}{q^{\alpha}}(-1)^{k} \frac{d^{k}}{d q^{k}}\left(e^{-q} q^{k+\alpha}\right) .
$$

We also note, for later use, that [4]

$$
\begin{gathered}
p_{k}^{\alpha}(q)=\sum_{r=0}^{k}\left(\begin{array}{l}
k \\
r
\end{array}\right)(-1)^{r} \frac{\Gamma(k+\alpha+1)}{\Gamma(k+\alpha-r+1)} q^{k-r} \\
\int_{0}^{\infty} d q e^{-q} q^{\alpha} p_{k_{1}}^{\alpha}(q) p_{k_{2}}^{\alpha}(q)=\Gamma\left(k_{1}+1\right) \Gamma\left(k_{1}+\alpha+1\right) \delta_{k_{1} k_{2}}
\end{gathered}
$$

$$
\int_{0}^{\infty} d q q^{a-1} e^{-q} p_{k}^{b}(q)=(1-a+b)_{k} \Gamma(a)(-1)^{k}
$$

recalling that, $(1-a+b)_{k}=(1-a+b)(1-a+b+$ 1) $\ldots(1-a+b+k-1)$. Writing $\Delta\left(q_{1}, \ldots, q_{m}\right)$ in terms of $p_{k}^{\alpha}(q)$ as in Eq. 1 and using the orthogonal property of these polynomials it immediately follows that:

$$
\begin{aligned}
S_{m n}= & \psi(m n+1) \\
& -\frac{1}{m n} \sum_{k=0}^{m-1} \int_{0}^{\infty} \frac{e^{-q}(q \ln q) q^{n-m}\left(p_{k}^{m-n}(q)\right)^{2} d q}{\Gamma(k+1) \Gamma(k+1+n-m)} .
\end{aligned}
$$

We thus need to evaluate the integral

$$
I_{n m}^{k}=\int_{0}^{\infty}(q \ln q) q^{n-m}\left(p_{k}^{m-n}(q)\right)^{2} e^{-q} d q .
$$

We first introduce

$$
J^{k}(\alpha)=\int_{0}^{\infty} q^{\alpha+1}\left(p_{k}^{\alpha}(q)\right)^{2} e^{-q} d q .
$$

This integral is easily evaluated. We have

$J^{k}(\alpha)=\Gamma(k+1) \Gamma(k+\alpha+2)+k^{2} \Gamma(k) \Gamma(k+\alpha+1)$

and we now note that

$$
I_{n m}^{k}=\left[\frac{d J^{k}(\alpha)}{d \alpha}-2 \int_{0}^{\infty} d q q^{\alpha+1} e^{-q} p_{k}^{\alpha} \frac{d p_{k}^{\alpha}}{d \alpha}\right]_{\alpha=n-m} .
$$

Evaluating these two terms using Eqs. (2), (3), (4), and (5) we find

$$
\begin{aligned}
S_{m n}= & \psi(m n+1) \\
& -\frac{1}{m n} \sum_{k=0}^{m-1}[1+(1+2 k+n-m) \psi(k+n-m+1)] \\
& +\frac{2}{m n} \sum_{k=0}^{m-1} \sum_{r=0}^{k}\left(\begin{array}{l}
k \\
r
\end{array}\right)(-1)^{k+r} \frac{\Gamma(k+n-m+1)}{\Gamma(k+n-m-r+1)} \\
& \times[\psi(k+n-m+1)-\psi(k+n-m-r+1)] \\
& \times \frac{(r-k-1)_{k} \Gamma(k+n-m-r+2)}{\Gamma(k+1) \Gamma(k+n-m+1)}
\end{aligned}
$$

where we use the fact that $\psi(z)=\frac{1}{\Gamma(z)} \frac{d \Gamma(z)}{d z}$. We now observe that

$$
\begin{aligned}
& \psi(m n+1) \\
& -\frac{1}{m n} \sum_{k=0}^{m-1}[1+(1+2 k+n-m) \psi(1+k+n-m)] \\
& =\sum_{k=n+1}^{m n} \frac{1}{k}+\frac{(m-1)}{2 n} .
\end{aligned}
$$

This follows by examining the coefficient of $\frac{1}{r}$ in

$$
\sum_{k=0}^{m-1}(1+2 k+n-m) \psi(1+k+n-m)
$$

and writing

$$
\psi(1+k+n-m)=-C+\sum_{r=1}^{k+n-m} \frac{1}{r} .
$$

The third expression in Eq. (6) above is

$$
\begin{aligned}
& \frac{2}{m n} \sum_{k=0}^{m-1} \sum_{r=0}^{k}\left(\begin{array}{l}
k \\
r
\end{array}\right)(-1)^{k+r} \frac{\Gamma(k+n-m+1)}{\Gamma(k+n-m-r+1)} \\
& \times[\psi(k+n-m+1)-\psi(k+n-m-r+1)] \\
& \times \frac{(r-k-1)_{k} \Gamma(k+n-m-r+2)}{\Gamma(k+1) \Gamma(k+n-m+1)} \\
& =\frac{2}{m n} \sum_{k=0}^{m-1}\left(\begin{array}{l}
k \\
1
\end{array}\right)(-1)^{2 k+1} \\
& =-2 \frac{(m-1)}{2 n} .
\end{aligned}
$$

Since $(r-k-1)_{k}=0$, for all $r \neq 0$ and $r \neq 1$, and also $\psi(k+n-m+1)-\psi(k+n-m-r+1)=0$ when $r=0$, we obtain

$$
S_{m n}=\sum_{k=n+1}^{m n} \frac{1}{k}-\frac{(m-1)}{2 n}
$$

as conjectured by Page.

This work is part of project $\mathrm{SC} / 218 / 94$ supported by Forbairt.

Electronic address: sen @ maths.tcd.ie

[1] D. N. Page, Phys. Rev. Lett. 71, 1291 (1993).

[2] E. Lubkin, J. Math. Phys. 19, 1028 (1978).

[3] S. Lloyd and H. Pagels, Ann. Phys. (N. Y.) 188, 186 (1988).

[4] A. P. Prudnikov, Yu. A. Brychkov, and O. I. Marichev, Integrals and Series Vol.2, Gordon and Breach Publishers (1988). 